\title{
Binding Energy of an Off-Center Hydrogenic Donor Impurity in a Finite Confining Potential Quantum Well
}

\author{
I.F.I. MIKHAIL AND I.M.M. ISMAIL \\ Department of Mathematics, Faculty of Science, Ain Shams University \\ Cairo, Egypt
}

(Received January 3, 2007; revised version April 18, 2007)

\begin{abstract}
The binding energy of an off-center hydrogenic donor impurity in a finite confining potential quantum well was calculated by using a variational method of the Bastard type. New analytical expressions for the binding energy and for the initial deviation from the result of an infinite confining potential well were derived for the ground energy state. The sign of the initial deviation was found to depend on the location of the impurity. The analytical expressions were utilized further to derive some other important parameters.
\end{abstract}

PACS numbers: 71.15.--m, 71.55.--i

\section{Introduction}

Low dimensional semiconductor heterostructures such as quantum wells, wires, boxes, and dots have gained a great importance in the last two decades due to their fundamental properties and their wide range of applications. Special care has been given to investigate these quantum systems in the presence of shallow impurities [1-9]. The problem of calculating the binding energy of hydrogenic impurity in a quantum well has been considered in the pioneering work of Bastard [10]. He studied the case of center and off-center impurity but in an infinite confining potential quantum well. He applied a variational approach that was based on modifying the exact wave function of an infinite quantum well by adding a term that represents the hydrogenic impurity. The resulting trial wave function depends on one variational parameter. The case of finite confining potential quantum well was dealt with by using a similar approach by Chaudhuri and Bajaj [11] and Elabsy [12]. Jia-Lin Zhu [13] and Greene and Bajaj [14, 15] applied more complicated variational methods. However, in all these treatments the case of a 
center impurity had only been considered. Also, Mailhiot et al. [16, 17] considered the case of finite confining potential quantum well by using a trial wave function which was expanded in terms of Gaussian type orbitals functions in the presence and in the absence of the impurity. They took into consideration the effect of the different masses and dielectric constants of the well and the barrier. The latter was dealt with by using a series of images. Their treatment was extended in a more accurate manner by Fraizzoli et al. [18], where a different basis for the expansion of the trial wave functions was used and the results were expressed in terms of integrals of an auxiliary variable. In both treatments a number of variational and fitting parameters were used and accordingly no final analytical expressions were derived. Further, analytical treatments have been performed recently (Blumel [19], Leyronas and Combescot [20]) to determine the energy eigenvalues of a finite potential quantum well in the absence of the impurity. This seems to be important, in spite of the fact that these eigenvalues have been obtained in all previous works by solving numerically the transcendental equation which results from the boundary conditions. At least, the analytical solution gives a useful guide to examine the accuracy of numerical results.

The case of off-center impurity in a finite confining potential well was dealt with by using a similar approach to that of Bastard [10] by Tanaka et al. [21] and Oliveira [22]. However, their procedure was entirely numerical and no analytical expressions similar to those obtained by Bastard [10] were derived. We believe that the derivation of analytical expressions for the energy expectation value and for the normalization constant are very useful in studying some important aspects of the problem. This is the main motivation of the present work. To the best of our knowledge the analytical results obtained here have not been published anywhere before. The numerical results of Greene and Bajaj [14] showed that the binding energy decreases linearly with $1 / \sqrt{V_{0}}$ for a center impurity and high values of the confining potential $V_{0}$ ( $V_{0}$ is much greater than the effective Rydberg). In the present work, we have derived an analytical expression for the initial deviation from the binding energy of infinite confining potential. The deviation varies linearly with $1 / \sqrt{V_{0}}$ in agreement with the results of Greene and Bajaj [14] for the center impurity. It has been shown further that the sign of this deviation depends on the position of the impurity. The analytical expressions obtained in the present work were employed to determine the position at which the deviation changes its sign. Moreover, these expressions were utilized to determine the width of the well which gives the maximum binding energy for different confining potentials and different locations of the impurity. The prediction of the width of the well which gives the maximum binding energy beforehand seems from our point of view to have important experimental applications.

The main purpose of the present work is to deal with the problem of off-center hydrogenic impurity in a finite confining potential quantum well by using a Bastard modified variational method. The present treatment is distinct 
from previous treatments [21, 22] by the derivation of the analytical expressions which are analogous to those obtained by Bastard [10] for the simple case of infinite confining potential. These expressions were then employed to study some important parameters. We believe that these parameters have significant effects in studying quantum wells with off-center impurities. The calculation of these parameters is thus a good advantage of the present variational method since it is difficult to calculate them with the more complicated wave functions used in other treatments. This is one of the reasons that we have restricted the calculations in the present article to the case of equal masses and dielectric constants for the well and the barrier. The other reason which seems to be more important is that it is preferable to use a trial variational wave function which resembles the exact solution in its main features, at least it should satisfy the same boundary conditions. The Bastard type of variational wave functions satisfies the required conditions only in the case of equal masses and accordingly the calculations have been restricted to this case. The problem of different masses will be considered in a future article by using an extended more complicated variational wave function.

The work is arranged in the following way. In Sects. 2, 3 the analytical expressions for the binding energy and for the initial deviation from the case of an infinite confining potential are derived. In Sect. 3, we have further presented the methods of determining the other parameters referred to above. In Sect. 4, the numerical results obtained in the case of $\mathrm{GaAs}-\mathrm{Ga}_{1-x} \mathrm{Al}_{x} \mathrm{As}$ are displayed.

\section{Ground impurity level in a quantum well}

In the existence of a hydrogenic impurity in a quantum well the Hamiltonian operator is given by

$$
H=\frac{p^{2}}{2 m^{*}}-\frac{e^{2}}{\epsilon\left|\boldsymbol{r}-\boldsymbol{r}_{0}\right|}+V(z)
$$

where the barrier potential $V(z)$ is taken to be a square well of height $V_{0}$ and width $L$,

$$
V(z)= \begin{cases}0 & \text { for }|z|<L / 2 \\ V_{0} & \text { for }|z|>L / 2\end{cases}
$$

Here we have assumed that the hydrogenic donor impurity is located at the point of position $\boldsymbol{r}_{0}$ with respect to the center of the well. Then, the position of the electron relative to the impurity is denoted by $\left|\boldsymbol{r}-\boldsymbol{r}_{0}\right|$ where $r=\sqrt{\rho^{2}+z^{2}}, \rho$ being the distance in the $x y$-plane

$$
\left|\boldsymbol{r}-\boldsymbol{r}_{0}\right|=\left[\rho^{2}+\left(z-z_{0}\right)^{2}\right]^{1 / 2},
$$

where $z_{0}$ is the position of the impurity on the $z$-axis. The values of the effective mass $m^{*}$ and the static dielectric constant $\epsilon$ are assumed to be the same across the interface between the two semiconductors.

In the absence of the impurity, the eigenfunction of the ground state is given by 


$$
\phi= \begin{cases}A \exp \left(k_{b}\left(z+\frac{L}{2}\right)\right) & \text { for } z \leq-L / 2, \\ N \cos \left(k_{\omega} z\right) & \text { for }|z| \leq L / 2, \\ B \exp \left(-k_{b}\left(z-\frac{L}{2}\right)\right) & \text { for } z \geq L / 2\end{cases}
$$

where $k_{\omega}, k_{b}$ are related to the eigenenergies $E$ and $V_{0}$ by

$$
E=\frac{\hbar^{2} k_{\omega}^{2}}{2 m^{*}}, \quad V_{0}-E=\frac{\hbar^{2} k_{b}^{2}}{2 m^{*}} .
$$

The application of the boundary conditions at $z= \pm L / 2$ gives

$$
B=A=N \cos \left(\frac{k_{\omega} L}{2}\right), \quad k_{b}=k_{\omega} \tan \left(\frac{k_{\omega} L}{2}\right) .
$$

The values of $k_{\omega}$ and $E$ can be obtained by solving numerically the transcendental Eq. (6).

In the presence of the impurity, we shall use a trial wave function of the following form:

$$
\psi=\phi \exp \left(-\frac{1}{\lambda}\left|\boldsymbol{r}-\boldsymbol{r}_{0}\right|\right),
$$

where $\lambda$ is a variational parameter and $\phi$ is defined from (4). The exponential term in (7) resembles the eigenfunction of the hydrogenic impurity in the ground state. Also, the trial wave function (7) satisfies the same boundary conditions as $\phi(\boldsymbol{r})$ and as the exact solution of the Hamiltonian operator $H$ defined by Eq. (1). It thus satisfies the condition of the continuity of the wave function and its gradient at $z= \pm L / 2$. Accordingly $A, B, N, k_{\omega}$, and $k_{b}$ are still related by Eq. (6). However, the normalization constant $(N)$ involved in (7) takes a very complicated form in comparison to that appearing in Eq. (4). After some laborious algebra, it is found that

$$
\begin{aligned}
N^{2}= & \frac{2}{\pi \lambda^{3}}\left\{\frac { 1 } { \lambda } \operatorname { c o s } ^ { 2 } ( \frac { k _ { \omega } L } { 2 } ) \frac { \mathrm { e } ^ { - L / \lambda } } { ( 1 + \lambda k _ { b } ) ^ { 2 } } \left[\left(\left(1+\lambda k_{b}\right)(L+\lambda)+\lambda\right)\right.\right. \\
& \left.\times \cosh \left(\frac{2 z_{0}}{\lambda}\right)-2\left(1+\lambda k_{b}\right) z_{0} \sinh \left(\frac{2 z_{0}}{\lambda}\right)\right]+1+g^{2} \cos \left(2 k_{\omega} z_{0}\right) \\
& -\mathrm{e}^{-L / \lambda} \cosh \left(\frac{2 z_{0}}{\lambda}\right)\left[1+\frac{L}{2 \lambda}+\frac{S Q g}{2 \lambda}+\frac{\lambda g k_{\omega}}{2} \sin \left(k_{\omega} L\right)\right] \\
& \left.+\left(\frac{z_{0}}{\lambda}\right) \mathrm{e}^{-L / \lambda} \sinh \left(\frac{2 z_{0}}{\lambda}\right)(1+S g)\right\}^{-1},
\end{aligned}
$$

where

$$
Q=L+2 \lambda g, \quad S=\cos \left(k_{\omega} L\right)-\lambda k_{\omega} \sin \left(k_{\omega} L\right), \quad g=\frac{1}{1+\lambda^{2} k_{\omega}^{2}} .
$$

Also, it can be shown after lengthy calculations that the expectation energy eigenvalue in the ground state is given by 


$$
\begin{gathered}
\varepsilon\left(L, z_{0}\right)=\langle\psi, H \psi\rangle=\frac{\hbar^{2} k_{\omega}^{2}}{2 m^{*}}+\frac{\hbar^{2}}{2 m^{*} \lambda^{2}}-\frac{e^{2} N^{2} \pi \lambda^{2}}{2 \epsilon}\left\{1-\mathrm{e}^{-L / \lambda} \cosh \left(\frac{2 z_{0}}{\lambda}\right)\right. \\
\left.+g \cos \left(2 k_{\omega} z_{0}\right)-\mathrm{e}^{-L / \lambda} \cosh \left(\frac{2 z_{0}}{\lambda}\right)\left[g S-\frac{1+\cos \left(k_{\omega} L\right)}{1+\lambda k_{b}}\right]\right\} .
\end{gathered}
$$

The variational parameter $\lambda$ can then be obtained by minimizing the energy $\varepsilon\left(L, z_{0}\right)$. The binding energy of the impurity is given by

$$
E_{\mathrm{b}}\left(L, z_{0}\right)=\frac{\hbar^{2} k_{\omega}^{2}}{2 m^{*}}-\varepsilon_{\min }\left(L, z_{0}\right)
$$

We note that the binding energy $E_{\mathrm{b}}\left(L, z_{0}\right)$ is a function of the layer thickness for a given $z_{0}$ and also it is a function of the potential barrier which confines the carrier in the well.

In the case of infinite potential well $V_{0} \rightarrow \infty$ and accordingly $k_{b} \rightarrow \infty$, $k_{\omega}=\pi / L$ and $S=-1$. Equations (8) and (10) will then be identical with the results of Bastard [10]. Also, in the special case of a center impurity $\left(z_{0}=0\right)$ Eqs. (8), (10) take the form

$$
\begin{gathered}
N^{2}=\frac{2}{\pi \lambda^{3}}\left\{\frac{1}{\lambda} \cos ^{2}\left(\frac{k_{\omega} L}{2}\right) \frac{\mathrm{e}^{-L / \lambda}}{\left(1+\lambda k_{b}\right)^{2}}\left[\left(1+\lambda k_{b}\right)(L+\lambda)+\lambda\right]\right. \\
\left.+1+g^{2}-\mathrm{e}^{-L / \lambda}\left[1+\frac{L}{2 \lambda}+\frac{S Q g}{2 \lambda}+\frac{\lambda g k_{\omega}}{2} \sin \left(k_{\omega} L\right)\right]\right\}^{-1}, \\
\varepsilon(L, 0)=\frac{\hbar^{2} k_{\omega}^{2}}{2 m^{*}}+\frac{\hbar^{2}}{2 m^{*} \lambda^{2}} \\
\quad-\frac{e^{2} N^{2} \pi \lambda^{2}}{2 \epsilon}\left\{1-\mathrm{e}^{-L / \lambda}+g-\mathrm{e}^{-L / \lambda}\left[g S+\frac{1+\cos \left(k_{\omega} L\right)}{1+\lambda k_{b}}\right]\right\} .
\end{gathered}
$$

In spite of the fact that the case of off-center and center impurities have been studied by other authors by using a wave function of the form (7), neither the results in (8), (10) nor in (12), (13) have been reported before, as the procedure was performed in the previous work in a complete numerical manner.

\section{Some important results}

In the case of very large but finite confining potential $\left(V_{0}\right)$ the transcendental Eq. (6) yields the approximate solution

$$
k_{\omega}=k_{0}-k, \quad k_{0}=\frac{\pi}{L}, \quad k=\frac{\pi \hbar}{L^{2}} \sqrt{\frac{2}{m^{*} V_{0}}} .
$$

Substituting in (8), (10) and retaining only the linear terms in $k$ we find

where

$$
\varepsilon\left(L, z_{0}\right)=\varepsilon^{(0)}\left(L, z_{0}\right)-\frac{\hbar^{2}}{m^{*}} k_{0} k+\beta(\lambda) V_{0}^{-1 / 2},
$$

$$
\varepsilon^{(0)}\left(L, z_{0}\right)=\frac{\hbar^{2} k_{0}^{2}}{2 m^{*}}+\frac{\hbar^{2}}{2 m^{*} \lambda^{2}}-\frac{e^{2}}{\epsilon} \frac{A^{\prime}}{\lambda C^{\prime}},
$$




$$
\begin{aligned}
\beta(\lambda) & =\frac{\hbar \pi}{L^{2}} \sqrt{\frac{2}{m^{*}}} \frac{g_{0} e^{2}}{\lambda \epsilon}\left(\frac{A^{\prime} D^{\prime}}{C^{\prime 2}}-\frac{B^{\prime}}{C^{\prime}}\right), \\
A^{\prime}= & 1+g_{0} \cos \left(2 k_{0} z_{0}\right)-\lambda^{2} k_{0}^{2} g_{0} \mathrm{e}^{-L / \lambda} \cosh \left(\frac{2 z_{0}}{\lambda}\right), \\
B^{\prime}= & 2 z_{0} \sin \left(2 k_{0} z_{0}\right)+2 \lambda^{2} k_{0} g_{0} \cos \left(2 k_{0} z_{0}\right)+\lambda k_{0} Q_{0} \mathrm{e}^{-L / \lambda} \cosh \left(\frac{2 z_{0}}{\lambda}\right), \\
C^{\prime}= & 1+g_{0}^{2} \cos \left(2 k_{0} z_{0}\right)+\frac{1}{2 \lambda} \mathrm{e}^{-L / \lambda} \cosh \left(\frac{2 z_{0}}{\lambda}\right)\left(g_{0} Q_{0}-L-2 \lambda\right) \\
& +z_{0} \lambda g_{0} k_{0}^{2} \mathrm{e}^{-L / \lambda} \sinh \left(\frac{2 z_{0}}{\lambda}\right), \\
D^{\prime}= & 2 z_{0} g_{0} \sin \left(2 k_{0} z_{0}\right)+4 \lambda^{2} k_{0} g_{0}^{2} \cos \left(2 k_{0} z_{0}\right)-z_{0} k_{0} Q_{0} \mathrm{e}^{-L / \lambda} \sinh \left(\frac{2 z_{0}}{\lambda}\right) \\
& +k_{0} \mathrm{e}^{-L / \lambda} \cosh \left(\frac{2 z_{0}}{\lambda}\right)\left[2 \lambda Q_{0} g_{0}+\frac{L(L+\lambda)}{2}\right],
\end{aligned}
$$

$\varepsilon^{(0)}\left(L, z_{0}\right)$ is the expectation energy eigenvalue in the case of infinite confining potential (Bastard [10]), $g_{0}$ is defined from (9) but with $k_{\omega}=k_{0}$ and $Q_{0}$ takes the same form as $Q$ after replacing $g$ by $g_{0}$.

If $\lambda_{0}, \lambda_{1}=\lambda_{0}+\delta \lambda$ are the values of $\lambda$ which minimize $\varepsilon^{(0)}\left(L, z_{0}\right)$ and $\varepsilon\left(L, z_{0}\right)$ in Eq. (15) then

$$
\left(\frac{\partial \varepsilon^{(0)}}{\partial \lambda}\right)_{\lambda=\lambda_{0}}=0, \quad \delta \lambda=-\left(\frac{\partial \beta}{\partial \lambda} / \frac{\partial^{2} \varepsilon^{(0)}}{\partial \lambda^{2}}\right)_{\lambda=\lambda_{0}} V_{0}^{-1 / 2}
$$

Thus, considering only the linear terms in $k$ (in $V_{0}^{-1 / 2}$ ) we find from (15) that

$$
\varepsilon_{\min }\left(L, z_{0}\right)=\varepsilon_{\min }^{(0)}\left(L, z_{0}\right)-\frac{\hbar^{2}}{m^{*}} k_{0} k+\beta\left(\lambda_{0}\right) V_{0}^{-1 / 2}
$$

and accordingly for large confining potential

where

$$
E_{\mathrm{b}}\left(L, z_{0}\right)=E_{\mathrm{b}}^{(0)}\left(L, z_{0}\right)-\beta\left(\lambda_{0}\right) V_{0}^{-1 / 2},
$$

$$
\varepsilon_{\min }\left(L, z_{0}\right)=\left.\varepsilon\left(L, z_{0}\right)\right|_{\lambda=\lambda_{1}}, \quad \varepsilon_{\min }^{(0)}\left(L, z_{0}\right)=\left.\varepsilon^{(0)}\left(L, z_{0}\right)\right|_{\lambda=\lambda_{0}}
$$

and $E_{\mathrm{b}}^{(0)}\left(L, z_{0}\right)$ is the corresponding binding energy for an infinite well. The result in (19) shows that the relation between $E_{\mathrm{b}}$ and $V_{0}^{-1 / 2}$ is linear for large $V_{0}$ such that $\beta\left(\lambda_{0}\right) \neq 0$. This result agrees with the findings of Greene and Bajaj [14] for a center impurity.

The results displayed in Fig. 1 show that $\beta\left(\lambda_{0}\right)$ (the - ve of the slope of the straight lines) is +ve for impurities located near the center while it is -ve for impurities located near the boundaries. This means that the initial deviation due to the consideration of finite potential barrier is either -ve or +ve, since it either decreases or increases the binding energy from the corresponding result of infinite confining potential. It is of importance to determine in an accurate manner the impurity position at which this deviation is equal to zero and accordingly the 

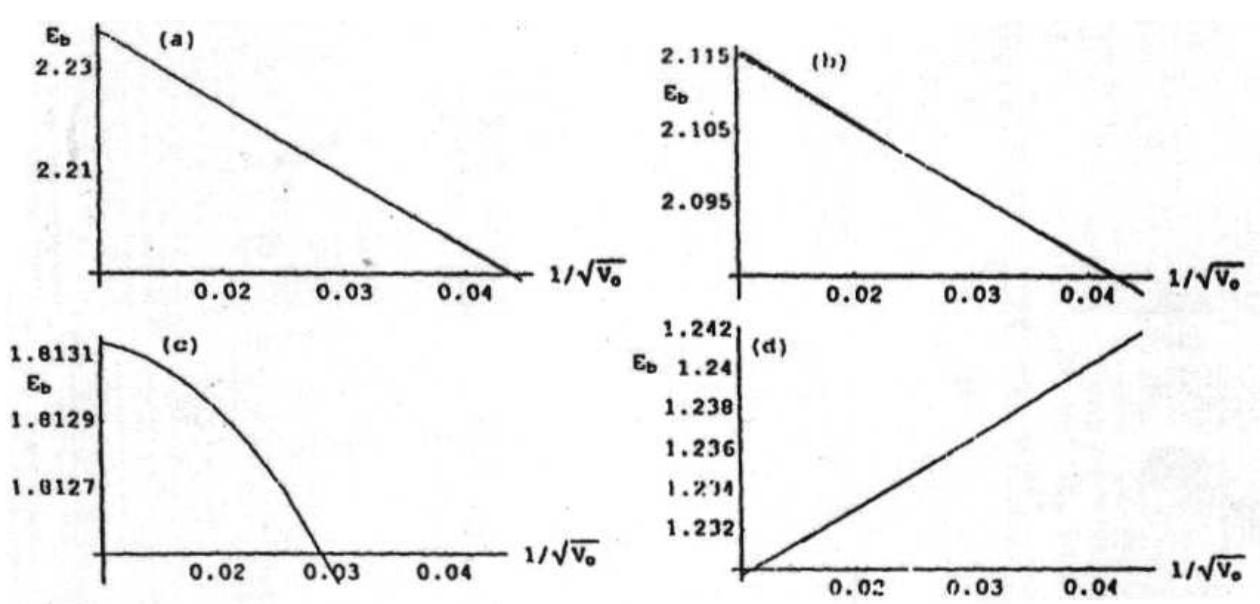

Fig. 1. A plot of the binding energy $E_{\mathrm{b}}$ versus $V_{0}^{-1 / 2}$ for $L=1$ and for (a) $z_{0}=0$, (b) $z_{0}=L / 8$, (c) $z_{0}=L / 4$, (d) $z_{0}=L / 2$.

critical value of $z_{0}$ at which the deviation changes its sign. This can be performed at large $V_{0}$ by solving the two equations

$$
\frac{\partial \varepsilon^{(0)}}{\partial \lambda}=0, \quad \beta(\lambda)=0
$$

to find both $\lambda_{0}$ and $z_{0}$.

It is also possible to determine the value of $z_{0}$ at any confining potential not necessarily large. In order to perform this procedure we have to replace the variational parameter $\lambda$ in the Bastard expression $\varepsilon^{(0)}\left(L, z_{0}\right)$ by $\mu$. Then for certain $L, V_{0}$, the equations

$$
\frac{\partial \varepsilon\left(L, z_{0}, \lambda\right)}{\partial \lambda}=0, \quad \frac{\partial \varepsilon^{(0)}\left(L, z_{0}, \mu\right)}{\partial \mu}=0, \quad \varepsilon\left(L, z_{0}, \lambda\right)=\varepsilon^{(0)}\left(L, z_{0}, \mu\right)
$$

have to be solved to find $\lambda, \mu, z_{0}$. The derivatives in (20), (21) have been performed algebraically by using the algebraic facilities of the Mathematica packages. They are not given explicitly here due to their length. The value of $z_{0}$ determines the required critical position of the impurity $z_{0 \mathrm{c}}$. From the physical point of view $z_{0 \mathrm{c}}$ stands for the location of the impurity at which the effect of finite confining potential on the binding energy is entirely obliterated. Thus, in this respect the well behaves as if it is infinitely confined.

It is clear from the results displayed in Fig. 2 that for a finite confining potential the binding energy increases as $L$ decreases until it reaches a maximum value at a certain well width $L_{\mathrm{m}}$. In fact, as the well width decreases, both the Coulomb interaction and the tunnelling effect increase. The former increases the binding energy while the latter decreases it. At $L=L_{\mathrm{m}}$ some kind of balance occurs between the two mechanisms and accordingly the binding energy is maximum. It is clearly desirable to determine $L_{\mathrm{m}}$ and the corresponding maximum 

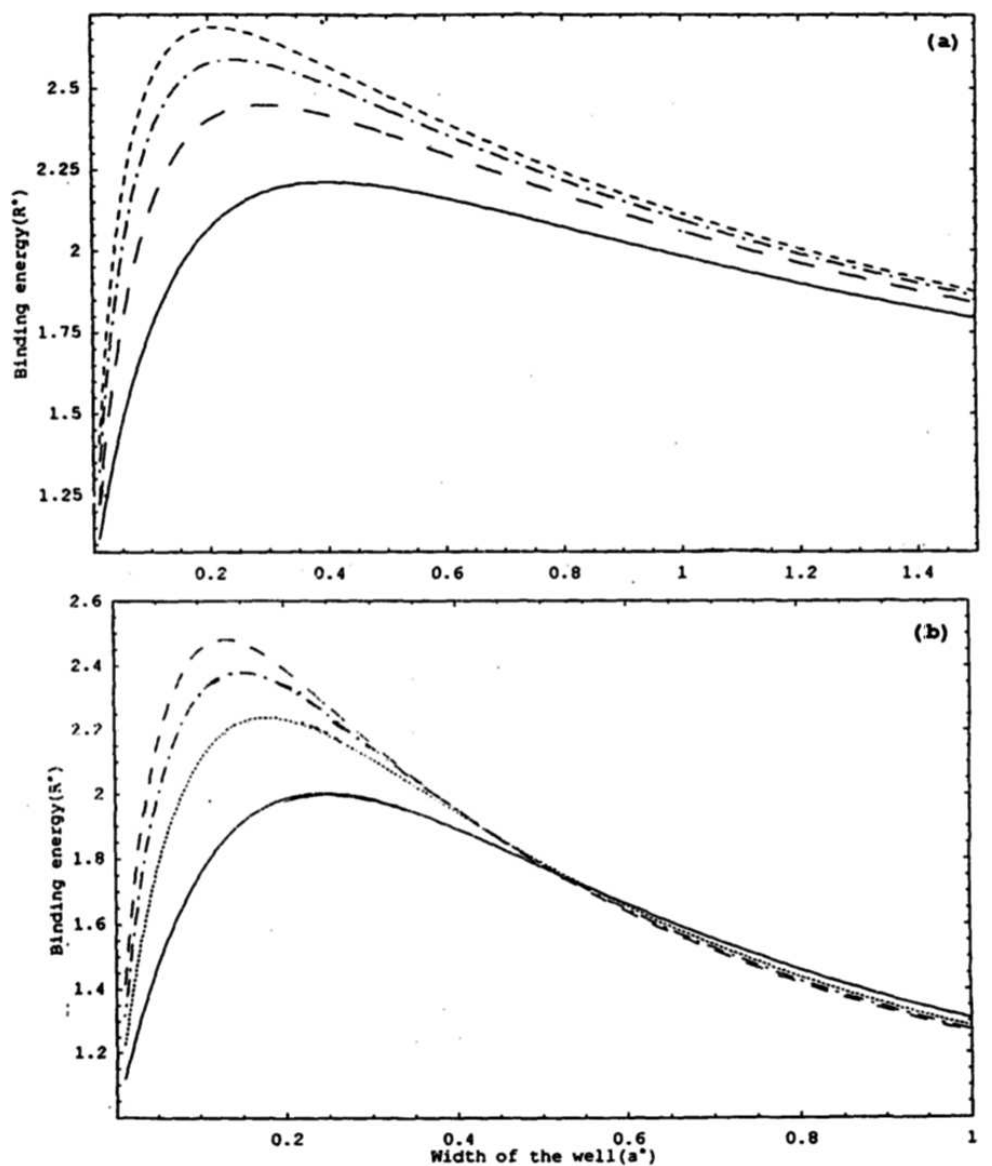

Fig. 2. Graph of the binding energy $E_{\mathrm{b}}$ against the width of the well $L$, for the center impurity $\left(z_{0}=0\right)(\mathrm{a})$ and for $z_{0}=L / 2(\mathrm{~b})$. The full, broken, dashed-dotted and dashed curves refer to $V_{0}=25,50,75,100 R^{*}$, respectively.

binding energy $E_{\mathrm{m}}$ for a certain $V_{0}$ and a certain impurity location $z_{0}$. For this purpose we put the transcendental Eq. (6) in the form

$$
L=\frac{2 x}{\sqrt{V_{0}} \cos (x)},
$$

where $x=k_{\omega} L / 2$ and therefore

$$
k_{\omega}=\frac{2 x}{L}=\sqrt{V_{0}} \cos (x), \quad k_{b}=\sqrt{V_{0}} \sin (x) .
$$

Consequently the expressions for $N^{2}$ and $\varepsilon\left(L, z_{0}\right)$ (Eqs. (8),(9)) depend only on $\lambda, x$. We thus take

$$
\frac{\partial \varepsilon}{\partial \lambda}=0, \quad \frac{\partial \varepsilon}{\partial x}=0
$$

and solve the resulting two equations to find the values of $\lambda$ and $x$. The corre- 
sponding value of $\varepsilon$ is minimum with respect to $\lambda$ and $x$ while the corresponding binding energy is maximum for both parameters. Also, the required value of $L$ is finally obtained from Eq. (22).

\section{Applications}

The results obtained in the previous two sections are applied to the case of GaAs- $\mathrm{Ga}_{1-x} \mathrm{Al}_{x}$ As quantum well heterostructure. According to Montenegro and Merchancano [23]:

$$
m^{*}=0.0665 m_{0}, \quad \epsilon=12.58\left(4 \pi \epsilon_{0}\right),
$$

where $m_{0}$ is the free electron mass and $\epsilon_{0}$ is the permittivity of free space. The energies and lengths in Figs. 1 to 3 are given respectively in units of the effective Rydberg $\left(R^{*}\right)$ and effective Bohr radius $\left(a^{*}\right)$, where

$$
R^{*}=\frac{m^{*} e^{4}}{2 \hbar^{2} \epsilon^{2}} \approx 5.72 \mathrm{meV}, \quad a^{*}=\frac{\hbar^{2} \epsilon}{m e^{2}} \approx 100 \AA .
$$

The relation between the binding energy $E_{\mathrm{b}}$ and the width of the well $L$ is displayed in Fig. 2 for different confining potentials $\left(V_{0}=25,50,75,100 R^{*}\right)$ and for a center impurity $\left(z_{0}=0\right)$ (Fig. $2 \mathrm{a}$ ) and for an impurity located at $z_{0}=L / 2$ (Fig. 2b). The general behavior of the results in Fig. 2a (center impurity) agrees with that of Greene and Bajaj [14]. The binding energy increases as $L$ decreases until it reaches a maximum value and then it starts to decrease. The width of the well can be decreased until it approaches zero, unlike the case of the spherical quantum dot where the dot, radius has a lower limit. The maximum values of the binding energy at $V_{0}=25,50 R^{*}$ are $2.21 R^{*}$ and $2.45 R^{*}$ which are very close to the values shown in Fig. 1 of Ref. [14].

The results in Fig. $2 \mathrm{~b}$ (off-center impurity, $z_{0}=L / 2$ ) behave in a similar manner. However, they are lower than the results in Fig. 2a (center impurity) in agreement with the fact that the center impurity possesses the highest binding energy.

The behavior of the results in Fig. 2a, b for a finite confining potential differs from that of an infinite confining potential (Bastard [10]). For an infinite confining potential the binding energy continues to increase as $L$ decreases until it tends to the ground state of the two-dimensional hydrogen atom at $L=0$ and accordingly the results do not exhibit a maximum value.

The variation of the binding energy with the position of the impurity $z_{0}$ is shown in Fig. 3 for $L=1$ and confining potentials $\left(V_{0}=50,100,150,200 R^{*}\right)$. The results (Fig. 3, main part) confirm the fact that the binding energy is maximum for the center impurity $\left(z_{0}=0\right)$, it decreases monotonically as $z_{0}$ increases. Moreover, the binding energy of impurities located near the center increases as the confining potential increases while it decreases for impurities located near the boundaries. This agrees with the conclusion that will be pointed out in the next section, regarding the sign of the initial deviation from the result of infinite confining potential. In the left bottom inset the equality of the binding energy for 


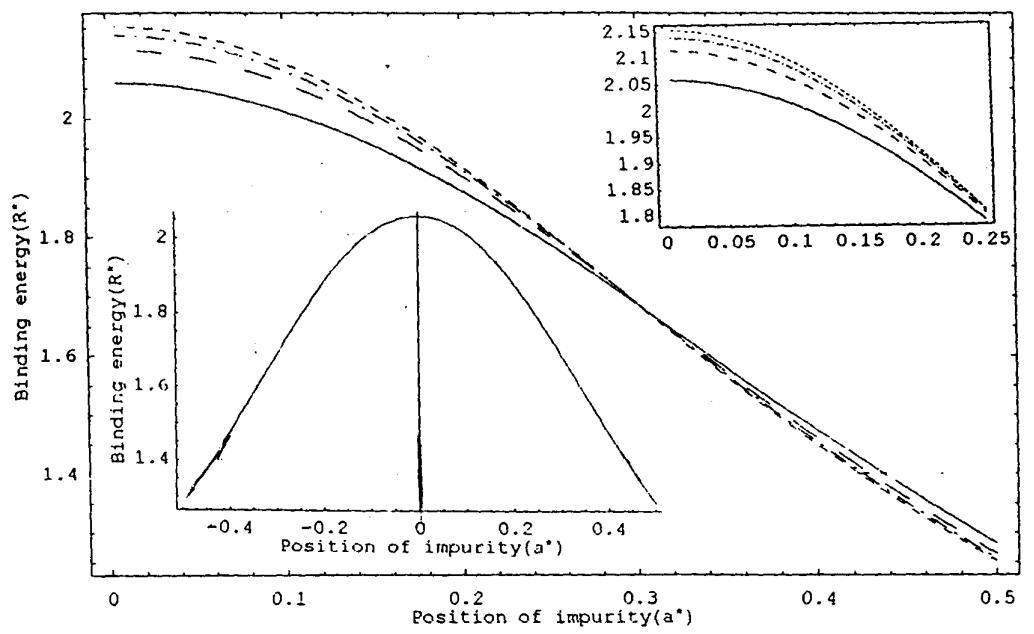

Fig. 3. Graph of the binding energy $E_{\mathrm{b}}$ against the position of the impurity $z_{0}$ at $L=1 a^{*}$. In the main part and in the right top inset the full, broken, dashed-dotted, and dashed curves refer to $V_{0}=50,100,150,200 R^{*}$, respectively. The right top inset shows the results up to $z_{0}=0.25 a^{*}$. In the left bottom inset the results are calculated at $V_{0}=50 R^{*}$ for + ve and - ve values of $z_{0}$.

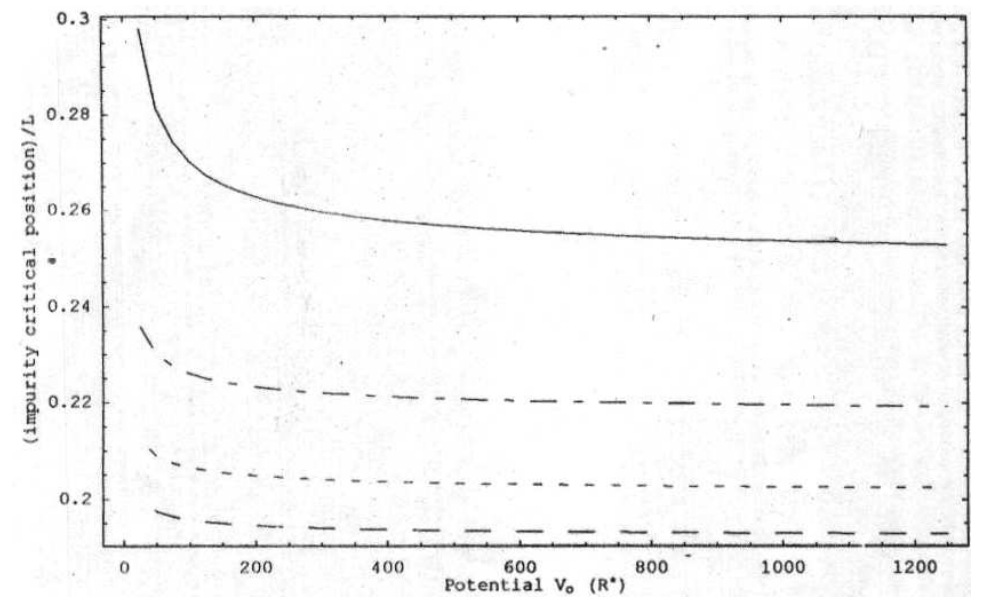

Fig. 4. Graph of the (impurity critical position) $/ L$ against the confining potential. The full, dashed-dotted, dotted, and broken curves stand for $L=1,2,3,4 a^{*}$, respectively.

impurities located at $\pm z_{0}$ is shown. The symmetry displayed in this figure can be directly concluded from Eqs. (8), (10). The results in Fig. 3 have the same trend as in the case of infinite confining potential (Bastard [10]).

Figure 1 shows the variation of $E_{\mathrm{b}}$ with $V_{0}^{-1 / 2}$ for large $V_{0}$. The results have been obtained from Eq. (11). For all values of $z_{0}$ considered in Fig. 1 the relation between $E_{\mathrm{b}}$ and $V_{0}^{-1 / 2}$ is linear except for $z_{0} \approx L / 4$. The slope of the straight 


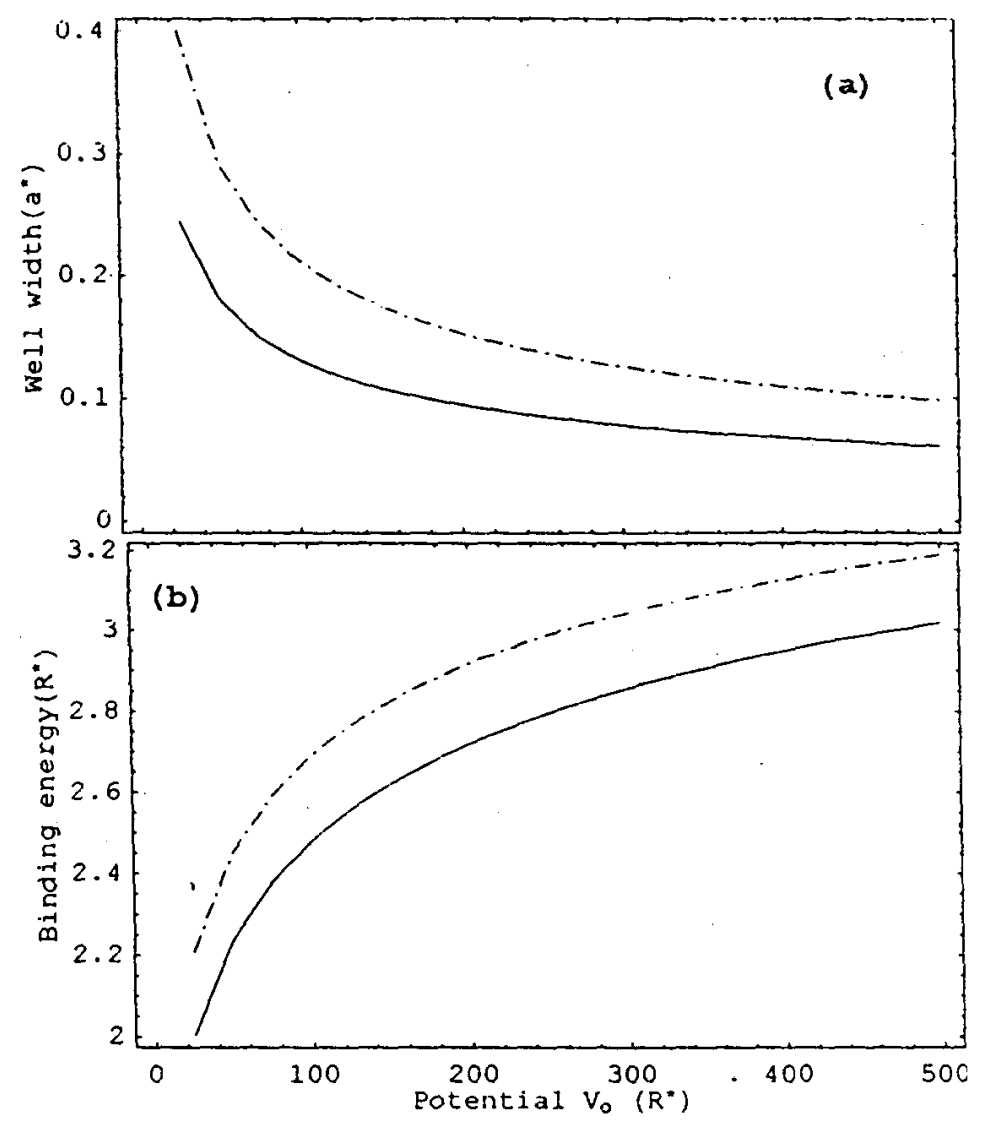

Fig. 5. Parts $(\mathrm{a}, \mathrm{b})$ show the well width which gives the maximum binding energy and the maximum binding energy, respectively. Here the dashed-dotted and full curves refer to $z_{0}=0, L / 2$, respectively.

lines agrees exactly with the value of $\beta\left(\lambda_{0}\right)$ calculated from (16). Also, these lines intersect the $E_{\mathrm{b}}$-axis at values which are identical with the corresponding values of $E_{\mathrm{b}}^{(0)}\left(L, z_{0}\right)$. It is further found that $\beta\left(\lambda_{0}\right)=0$ at $z_{0} \approx L / 4$. For this reason the relation is quadratic for this particular value of $z_{0}$ rather than being linear. Moreover, for $z_{0}<L / 4, \beta\left(\lambda_{0}\right)>0$ while for $z_{0}>L / 4, \beta\left(\lambda_{0}\right)<0$. The present results thus lead to the conclusion that the application of a finite confining potential may initially decrease or increase the binding energy according to the position of the impurity.

The critical value of $z_{0}$ at which the initial deviation from the case of infinite well changes its sign has been calculated by using Eq. (21). At high values of $V_{0}$ the results have been checked by using Eq. (20). The results are shown in Fig. 4. For $L=1 a^{*}$ and high values of $V_{0}$ the deviation changes its sign at $z_{0} / L \approx 0.25$ in 
agreement with the results displayed in Fig. 1. Also, in Fig. 5a, b the well width $L$ at which the binding energy is maximum and the maximum binding energy are plotted against the confining potential $V_{0}$ at $z_{0}=0, L / 2$. The results have been calculated by utilizing Eqs. (24), (22).

\section{Conclusion}

The variational method applied in the present work has enabled us to derive an analytical expression for the binding energy of the impurity in the case of a finite confining potential well, unlike the variational methods used in the previous work, where the results were obtained numerically. The analytical approach has further been utilized to derive an expression for the first order deviation from the case of infinite confining potential well. The change of the location of the impurity affects substantially the order, sign, and behavior of this deviation. For a particular impurity position, the deviation varies quadratically with $V_{0}^{-1 / 2}$ in contrast to other locations, where it behaves linearly. This particular impurity position has further been determined at different confining potentials and well widths by utilizing the analytical expressions derived in the present work. Also, the well width which gives the maximum binding energy has been calculated.

\section{References}

[1] Jia-Lin Zhu, Sheng Xu, Phys. Rev. B 50, 12175 (1994).

[2] Z.X. Jiang, B.D. McCombe, Jia-Lin Zhu, W. Schaff, Phys. Rev. B 56, R1692 (1997).

[3] J.C.L. Cetina, N.P. Montenegro, Phys. Status Solidi B 210, 717 (1998).

[4] J.J.V. Moreno, N.P. Montenegro, Phys. Status Solidi B 210, 723 (1998).

[5] A. Zrenner, J. Chem. Phys. 112, 7790 (2000).

[6] D. Bimberg, N.N. Ledentsov, J. Phys., Condens. Matter 15, R1063 (2003).

[7] I.D. Mikhailov, F.J. Betancur, R.A. Escorcia, J.S. Ortega, Phys. Rev. B 67, 115317 (2003).

[8] R.T. Senger, K.K. Bajaj, Phys. Rev. B 68, 205314 (2003).

[9] P. Villamil, C. Cabra, N.P. Montenegro, J. Phys., Condens. Matter 17, 5049 (2005).

[10] G. Bastard, Phys. Rev. B 24, 4714 (1981).

[11] S. Chaudhuri, K.K. Bajaj, Phys. Rev. B 29, 1803 (1984).

[12] A.M. Elabsy, J. Phys., Condens. Matter 6, 10025 (1994).

[13] Jia-Lin Zhu, J. Phys., Condens. Matter 1, 1539 (1989).

[14] R.L. Greene, K.K. Bajaj, Solid State Commun. 45, 825 (1983).

[15] R.L. Greene, K.K. Bajaj, Phys. Rev. B 31, 4006 (1985).

[16] C. Mailhiot, Yia-Chung Chang, T.C. McGill, Phys. Rev. B 26, 4449 (1982).

[17] C. Mailhiot, Yia-Chung Chang, T.C. McGill, Surf. Sci. 113, 161 (1982).

[18] S. Fraizzoli, F. Bassani, R. Buczko, Phys. Rev. B 41, 5096 (1990). 
[19] R. Blumel, J. Phys. A, Math Gen. 38, L673 (2005).

[20] X. Leyronas, M. Combescot, preprint cond-mat/0201553, 2003 (see http//xxx.lanl.gov).

[21] K. Tanaka, M. Nagaoka, T. Yamabe, Phys. Rev. B 28, 7068 (1983).

[22] L.E. Oliveira, Phys. Rev. B 38, 10641 (1988).

[23] N.P. Montenegro, S.T.P. Merchancano, Phys. Rev. B 46, 9780 (1992). 